\title{
Trends in national and state-level obesity in the USA after correction for self-report bias: analysis of health surveys
}

\author{
Majid Ezzati ${ }^{1,2}$ Hilarie Martin ${ }^{2}$ Suzanne Skjold² Stephen Vander Hoorn ${ }^{3}$ Christopher J L Murray ${ }^{1,2}$
}

J R Soc Med 2006;99:250-257

\section{SUMMARY}

Objectives: To quantify population-level bias in self-reported weight and height as a function of age, sex, and the mode of selfreport, and to estimate unbiased trends in national and state level obesity in the USA.

Design: Statistical analysis of repeated cross-sectional health examination surveys (the National Health and Nutrition Examination Survey [NHANES]) and health surveys (the Behavioral Risk Factor Surveillance System [BRFSS]) in the USA.

Setting: The 50 states of the USA and the District of Columbia. Results: In the USA, on average, women underreported their weight, but men did not. Young and middle-aged ( $<65$ years) adult men over-reported their height more than women of the same age. In older age groups, over-reporting of height was similar in men and women. Population-level bias in self-reported weight was larger in telephone interviews (BRFSS) than in-person interviews (NHANES). Except in older adults, height was overreported more often in telephone interviews than in-person interviews. Using corrected weight and height in the year 2000, Mississippi (31\%) and Texas (30\%) had the highest prevalence of obesity for men; Texas (37\%), Louisiana (37\%), Mississippi (37\%), District of Columbia (37\%), Alabama (37\%), and South Carolina (36\%) for women.

Conclusions: Population-level bias in self-reported weight and height is larger in telephone interviews than in-person interviews. Telephone interviews are a low-cost method for regular, nationally- and sub-nationally representative monitoring of obesity. It is possible to obtain corrected estimates of trends and geographical distributions of obesity from telephone interviews by using systematic analysis which measure weight and height from an independent sample of the same population.

\section{INTRODUCTION}

Overweight and obesity are among the leading causes of mortality and morbidity, causing an estimated 2.6 million deaths worldwide and $2.3 \%$ of the global burden of

${ }^{1}$ Harvard School of Public Health, Boston; ${ }^{2}$ Initiative for Global Health, Harvard University, Cambridge, USA; ${ }^{3}$ Clinical Trials Research Unit, University of Auckland, New Zealand

Correspondence to: Majid Ezzati

E-mail: mezzati@hsph.harvard.edu disease ${ }^{1}$ they have increased in nearly all populations. ${ }^{2,3}$ Rising obesity as a cause of mortality has also been a subject of research and analysis in the USA. ${ }^{4,5}$ As a result, there is an unparalleled interest in national and sub-national monitoring of overweight and obesity, and on a regular basis. ${ }^{6-8}$

While technically straightforward, measuring weight and height in large nationally and sub-nationally representative samples and on a regular basis (e.g. annually) is costly. For this reason, population-level surveillance and health research regularly rely on self-reported weight and height. Self-reported weight and height data are subject to random error, and, more importantly, systematic reporting bias. $^{9-14}$ The magnitude of bias has varied across studies based on factors such as age, actual weight and height, and education. ${ }^{9}$ Some researchers have nonetheless concluded that self-reported height and weight are acceptable, valid, or excellent for population-based studies. ${ }^{11-14}$ The US Centers for Disease Control and Prevention (CDC), while acknowledging the bias in self-reported weight and height, presents state-level obesity levels and trends based on the Behavior and Risk Factor Surveillance Survey (BRFSS), which uses telephone surveys. ${ }^{7,8}$

In previous research, bias in self-reported height and weight has been characterized at the individual level, using measured and self-reported data from the same subjects. ${ }^{9-14}$ Subjects may, however, reduce intentional misreporting of their weights and heights, if they are measured before/after the interview. The 'mode' of interview (e.g. telephone versus in-person) can also affect misreporting as respondents may misreport less when in-person methods are used than in telephone interviews. The mode of interview may result in differential participation rates in different health surveys. Therefore, the total bias in self-reported weight and height at the population-level arises from two sources: first, bias in individual reporting behaviour; and, second, systematic differences in participation in different survey modes. Thus, the very data needed for individual-level validation would make the findings inapplicable to population-level data if based solely on self-reported weight and height, especially those given in telephone interviews. The solution to this apparent dilemma is to adjust selfreported weight and height using measured values at the population levels, with the two estimates obtained independently. 
We estimated the population-level relationship between measured and self-reported height and weight in the USA using two nationally representative health surveys and health examination surveys: the BRFSS and the National Health and Nutrition Examination Survey (NHANES). We also examined the role of age and sex on bias in selfreported weight and height. We used this relationship to correct self-reported weight and height from telephone surveys and to estimate the corrected trends in national and state-level obesity in the USA. In addition to providing the first unbiased estimates of the levels and trends in state-level obesity in the USA, this report contributes to methods for measurement and surveillance obesity, and other risks and diseases that regularly rely on self-report data, by quantifying the effects of the mode of self-report as a source of bias.

\section{METHODS}

\section{Data sources}

We used data from two nationally representative health surveys and health examination surveys, the BRFSS and NHANES, for two time periods (1988-1994 and 19992002). NHANES is conducted by the CDC, and includes a series of cross-sectional nationally representative health examination surveys beginning in 1960. The third NHANES (NHANES III) was conducted between 1988 and 1994. Beginning in 1999, NHANES became a continuous survey, with data for 1999-2002 available for analysis. In each survey a nationally representative sample of the US civilian non-institutionalized population was selected using a complex, stratified, multistage probability cluster sampling design. Self-reported weight and height were recorded from in-person interviews at home. Subsequently individuals were invited for a clinical examination in a mobile examination centre, or in their home if they are unable to travel. The response rate for the household interview in NHANES is $>80 \%$ and for medical examination $>75 \%$. Detailed descriptions of the survey methods, including weight and height measurement techniques, are available elsewhere ${ }^{10,15-17}$ and on-line [http://www.cdc.gov/nchs/ nhanes.htm].

The BRFSS is a cross-sectional telephone survey designed and managed by the CDC but administered by state health departments. The BRFSS uses a multistagecluster design based on random-digit dialing to select a representative sample from each state's non-institutionalized civilian residents aged 18 years or older. Data from each state are pooled to produce nationally representative estimates. The BRFSS questionnaire primarily focuses on personal risk behaviours and exposures. Median state overall response rate for the BRFSS in 2002 was 45\%; median Council of American Survey Research Organiza-
Table 1 Sample sizes for data sources used in the analysis. Sample sizes are for adults over the age of 20 years

\begin{tabular}{lrr}
\hline & \multicolumn{2}{c}{ Survey period } \\
\cline { 2 - 3 } Survey type & $\mathbf{1 9 8 8 - 1 9 9 4}$ & $\mathbf{1 9 9 9 - 2 0 0 2}$ \\
\hline NHANES & & \\
$\quad$ Total & 18825 & 10291 \\
M & 8816 & 4805 \\
F & 10009 & 5486 \\
Number with self-reported and & 15883 & 8841 \\
measured height and weight & & \\
BRFSS & & \\
$\quad$ Total & 597047 & 804913 \\
$\quad$ M & 250389 & 325804 \\
$\quad$ F & 346658 & 479109 \\
Number with self-reported height & 578207 & 768380 \\
and weight & & \\
\end{tabular}

NHANES, National Health and Nutrition Examination Survey; BRFSS, Behavioral Risk Factor Surveillance System.

tions response rate was $58 \%$. Detailed descriptions of the survey methods are available elsewhere ${ }^{18,19}$ and on-line [http://www.cdc.gov/brfss/].

\section{Statistical analysis}

NHANES household, interview, and examination data files were merged using the unique sequence number given to each participant. Subjects who did not participate in both the interview and the examination were excluded (Table 1). Samples were weighted using the procedure recommended in the BRFSS and NHANES documentation. Age-sexspecific (5 year age groups between 20 and 79 , and $80+$ ) mean population height, weight, and body mass index (BMI), defined as weight divided by height-squared $(\mathrm{kg} /$ $\mathrm{m}^{2}$ ), were calculated for both measured and self-reported variables for NHANES. For BRFSS, age-sex-specific mean population BMI, height and weight were calculated for each survey year corresponding to NHANES. Averages of BRFSS survey years corresponding to each NHANES round were used for comparison with NHANES.

\section{RESULTS}

\section{Bias in self-reported height and weight}

Figure 1 compares mean BMI, calculated using self-reported and measured weight and height, by age and sex for the two analysis periods. Except in men under 35 years of age in inperson interviews, self-reported weight and height systematically underestimated BMI compared to the 'gold standard' of measured health examination. Underestimation 

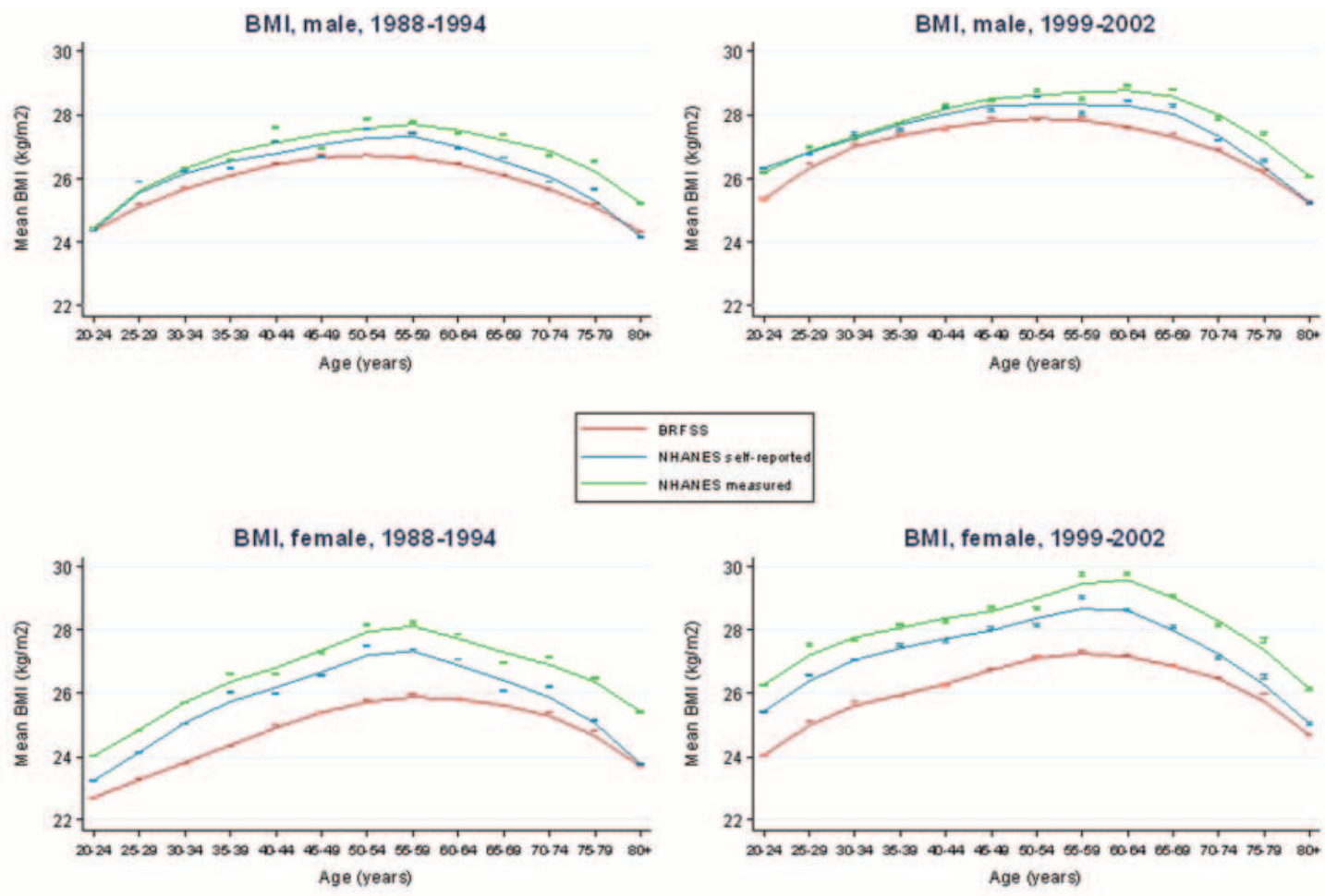

Figure 1 Measured (National Health and Nutrition Examination Survey [NAHNES]) and self-reported (NHANES in-person and Behavioral Risk Factor Surveillance System [BRFSS] telephone) BMI by age and sex. Each data point shows the $95 \%$ confidence interval (Cl) for estimated body mass index as two horizontal lines. When the $95 \% \mathrm{Cl}$ is small, the two horizontal lines appear to overlap. This is particularly the case for BRFSS (shown in red), which has very large sample size. For each survey, a local (non-parametric) regression was used to estimate the age pattern, shown as solid lines.

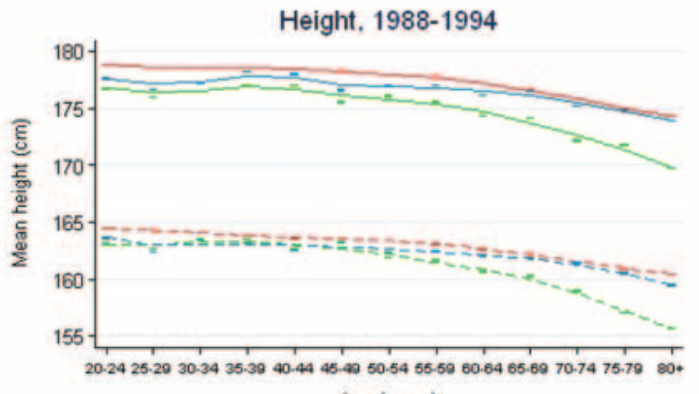

Age (years)

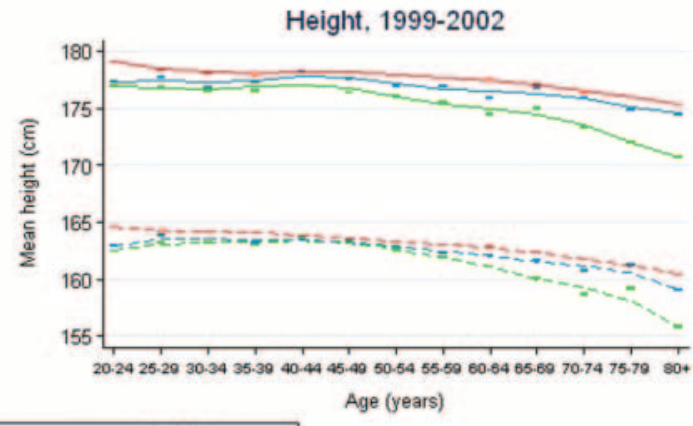

Age (years)
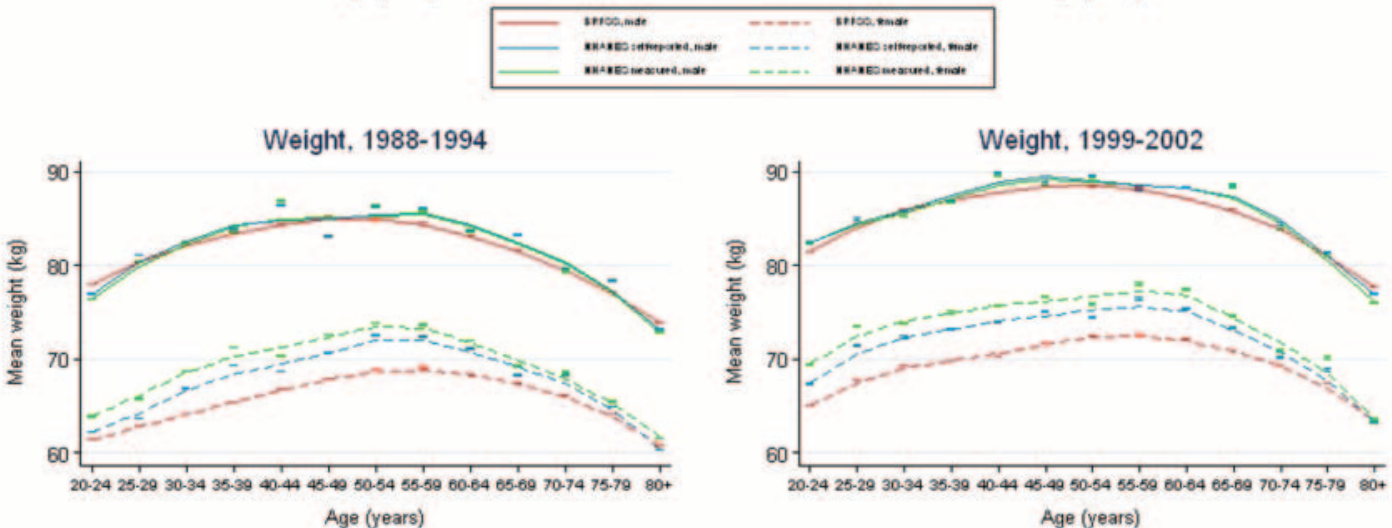

Figure 2 Measured (National Health and Nutrition Examination Survey [NAHNES]) and self-reported (NHANES in-person and Behavioral Risk Factor Surveillance System [BRFSS] telephone) height and weight by age and sex. Each data point shows the $95 \%$ confidence (CI) for estimated body mass index as two horizontal lines. When the $95 \% \mathrm{Cl}$ is small, the two horizontal lines appear to overlap. This is particularly the case for BRFSS (shown in red), which has very large sample size. For each survey, a local (non-parametric) regression was used to estimate the age pattern, shown as solid lines. 

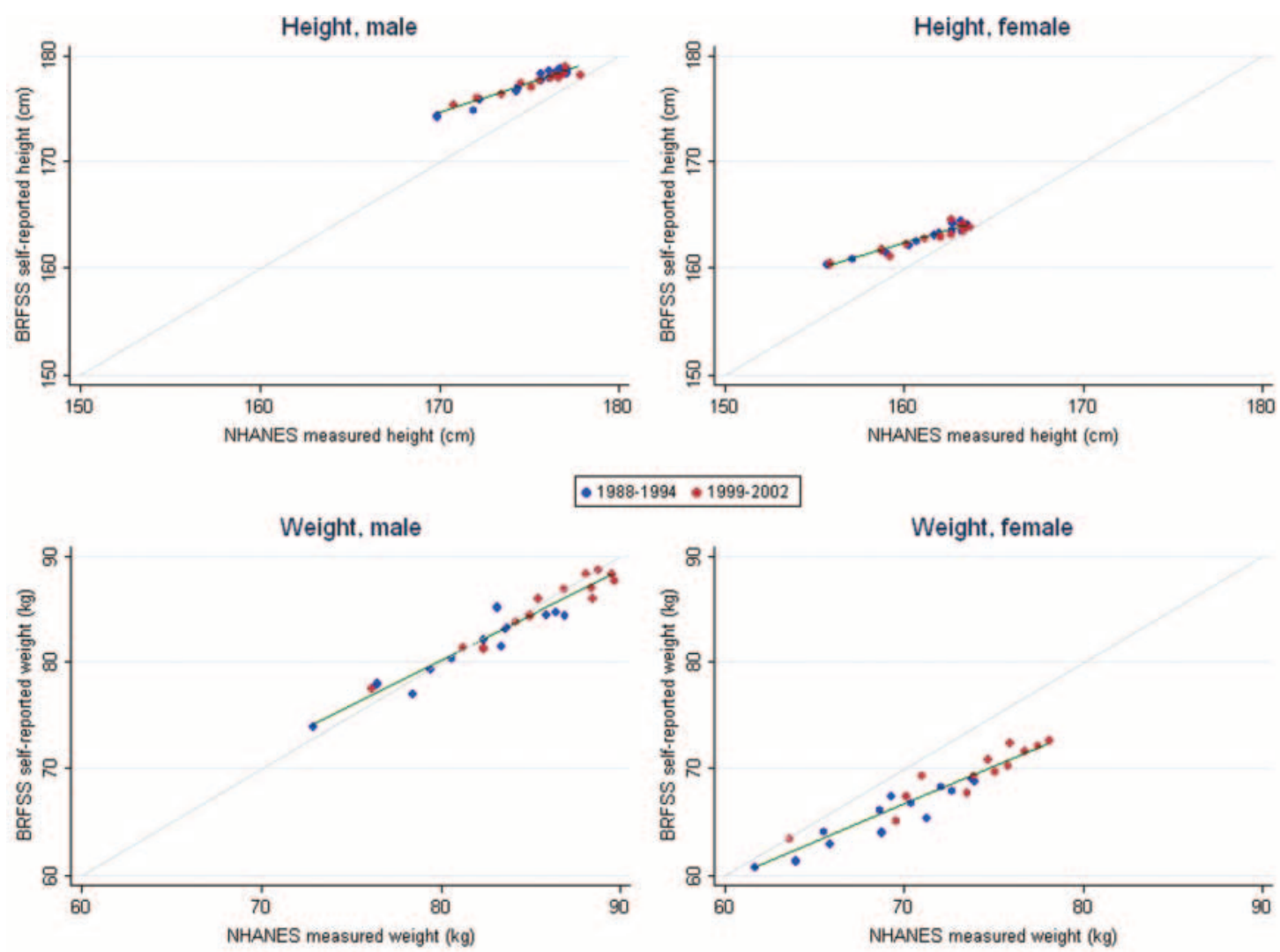

Figure 3 Self-reported height and weight in Behavioral Risk Factor Surveillance System (BRFSS) telephone surveys in relation to measured height and weight from National Health and Nutrition Examination Survey (NAHNES). Each point represents one 5-year age group (i.e. see the points in Figure 2), shown in blue for 1988-1994 and in red for 1999-2002
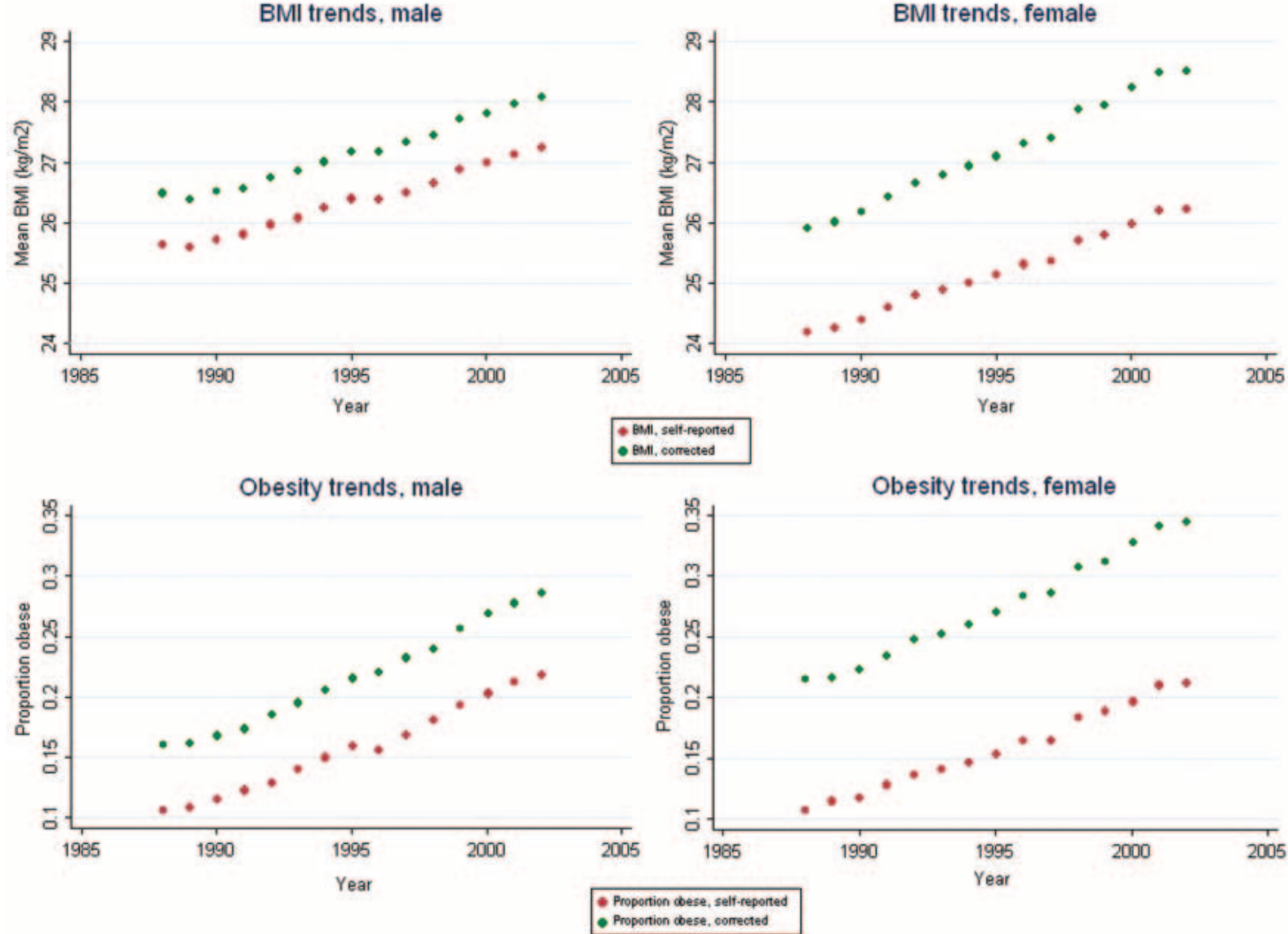

Figure 4 Trends in mean body mass index and obesity in men and women in the USA, based on self-reported Behavioral Risk Factor Surveillance System (BRFSS) height and weight as well as corrected height and weight. All values are age-standardized to the 2000 USA population 
was greater in telephone interviews than in-person interviews, especially among the 20-44 and 45-64 year age groups. The self-report bias was greater for women than for men, especially among the young and the middleaged. BMI underestimation may be caused by overestimating height, underestimating weight, or a combination of the two. Figure 2 shows that, on average, American men did not underestimate their weight in either in-person or telephone interviews, except in older ages. Weight for American women was underestimated, especially in the young and middle-aged ( $<65$ years), with larger underreporting in telephone interviews. Underestimation increased during the 1990 s, a period during which average weight increased, in absolute terms (from $3.74 \mathrm{~kg}$ in 1988 1994 to $4.39 \mathrm{~kg}$ in 1999-2002) and in relative terms (from $5.3 \%$ in $1988-1994$ to $5.9 \%$ in $1999-2002$ ).

Bias in self-reported height had a more complex pattern than that of weight. In younger ages (20-44 years), selfreported height was overestimated for both men and women, with larger overestimation for men than women, and in telephone interviews than in-person interviews. After this age, height was still overestimated, but overestimations for men and women, and in telephone and inperson interviews, gradually converged. The role of age in over-reporting height may be because height declines in older ages. If people measure their height less frequently than their weight, they may report measurements taken from early adulthood. This 'unintentional' misreporting would also explain the convergence of height estimates in telephone and in-person interviews.

Figure 3 plots the mean population height and weight from BRFSS against measured values from NHANES, by sex. The vertical deviation from the $45^{\circ}$ line measures bias in BRFSS data, as a function of those measured in NHANES. Figure 3 confirms the sex-specific results in Figure 2 (e.g. the absence of bias in self-reported weight among men because the BRFSS-NHANES plot coincides with the $45^{\circ}$ line). In addition, Figure 3 shows that the biases for all age groups, and in the two survey periods, are linear functions of measured height and weight from NHANES (i.e. those age groups with lower height or higher weight had large misreporting, proportional to their measured height or weight). This result is consistent with findings from individual-level analyses, which have shown that misreporting is a function of actual weight and height, generally leading to larger underestimation among those with higher BMI. ${ }^{9}, 10$

\section{Trends in national and state-level obesity in the USA}

We used the relationships in Figure 3 to correct individual self-reported height and weight from the BRFSS (which is conducted annually and is state-representative), and to estimate corrected BMI and obesity (defined as BMI $\geqslant 30$ ). The corrected values are those that would be expected if annual state-representative examination surveys such as NHANES had been conducted. Figure 4 shows that between 1988 and 2002, the corrected prevalence of obesity among adult Americans increased from 16.0\% to $28.7 \%$ for men and $21.5 \%$ to $34.5 \%$ for women, with a nearly linear trend (there is an apparent flattening in 2002 but definitive conclusions require data from subsequent years). In comparison, the prevalence of obesity was $19.7 \%$ for men and 24.5\% for women in NHANES III (19881994 ) and $26.6 \%$ for men and $32.7 \%$ for women in continuous NHANES (1999-2002) (if corrected BRFSS values are averaged over the same years, the values would be $17.9 \%$ for $1988-1994$ and $27.3 \%$ in $1999-2002$ for men and 23.6\% for 1988-1994 and 33.2\% in 19992002 for women). Figure 3 also shows that the difference between corrected and self-reported obesity showed a greater increase for women than for men, with selfreported obesity $6.8 \%$ for males and $13.3 \%$ for females lower than corrected values in 2002, versus 5.4 and 10.7 , respectively, in 1988.

Figure 5 compares the self-reported and corrected prevalence of obesity in the US states for 1990 and 2000. In 1990, self-reported obesity in all US states was below $18 \%$ for men and women. ${ }^{8}$ Corrected estimates show obesity prevalence $>18 \%$ in 14 states for men and in 44 states for women (including 11 states $>24 \%$ ). After correction, in 1990 , states with the highest prevalence of obesity for men were Mississippi (22\%), Hawaii (22\%) and Michigan (20\%) and for women District of Columbia (34\%), Delaware (27\%) and Mississippi (26\%); states with the lowest prevalence of obesity for men were Colorado (9\%), Utah (11\%) and Washington (12\%) and for women Massachusetts (17\%), Colorado (17\%) and Minnesota (18\%). In 2000 , self-reported obesity was below $24 \%$ in all but two states (Mississippi and Nebraska) for men and in all but three states (Alabama, Mississippi and District of Columbia) for women: these states had self-reported obesity prevalence between 24\% and 30\%. When height and weight were corrected for self-report bias, men in 39 states had obesity prevalence $>24 \%$, including two states with prevalence $\geqslant 30 \%$-Mississippi (30\%), and Texas (31\%); women in all states except Colorado had obesity prevalence $>24 \%$, including 33 states with prevalence $>30 \%$ and six states with prevalence $\geqslant 36 \%$ - the District of Columbia (37\%), Texas (37\%), Louisiana (37\%), Mississippi (37\%), Alabama (37\%) and South Carolina (36\%). States with the lowest prevalence of corrected obesity for men in 2000 were Colorado (18\%), District of Columbia (21\%) and Montana (21\%) and for women Colorado (24\%), Montana (16\%) and Massachusetts (27\%). 
Male, self-reported, 1990

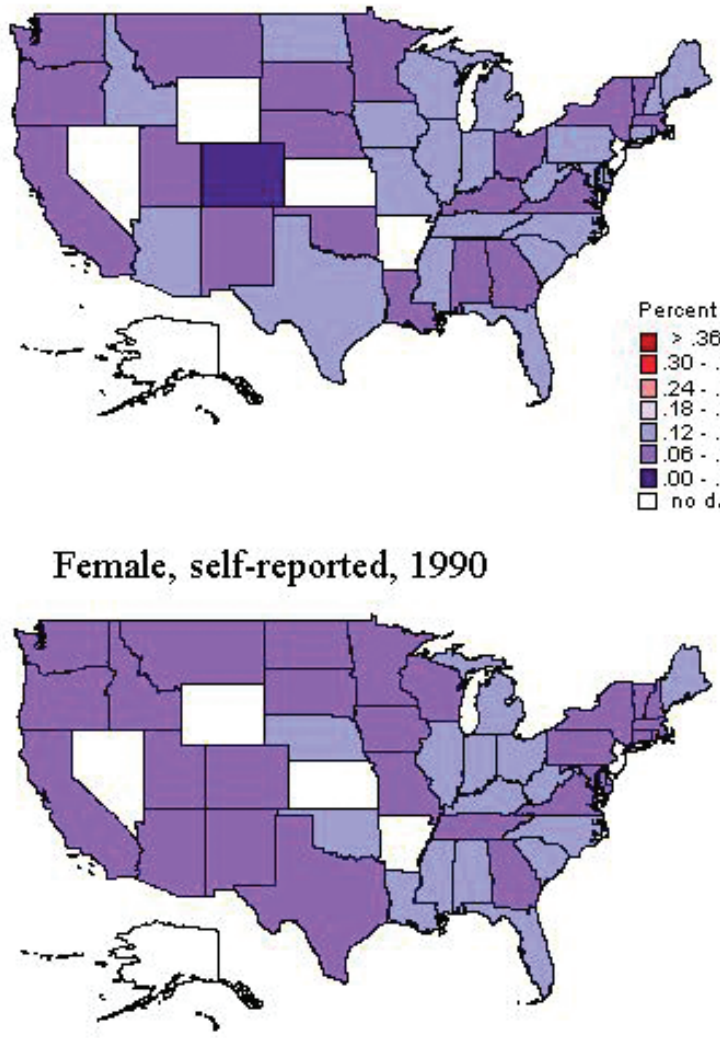

Male, self-reported, 2000

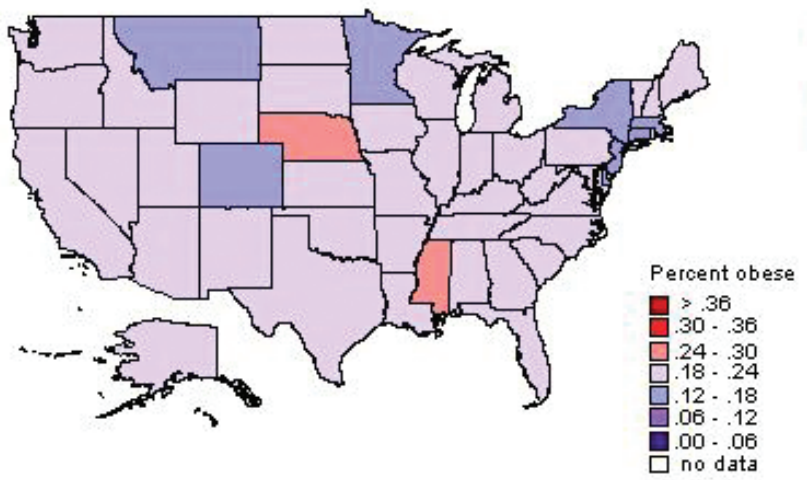

Female, self-reported, 2000

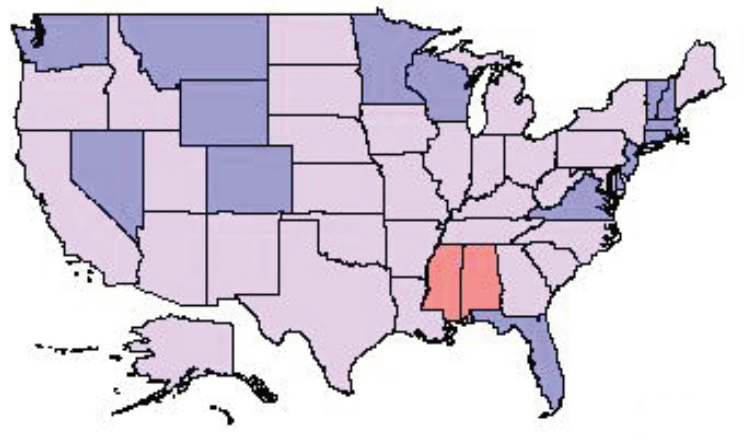

Figure 5 Prevalence of obesity in USA states in (a) 1990 and (b) 2000, based on self-reported Behavioral Risk Factor Surveillance System (BRFSS) height and weight as well as corrected height and weight. All values are age-standardized to the 2000 USA population.
Male, corrected, 1990

(a)

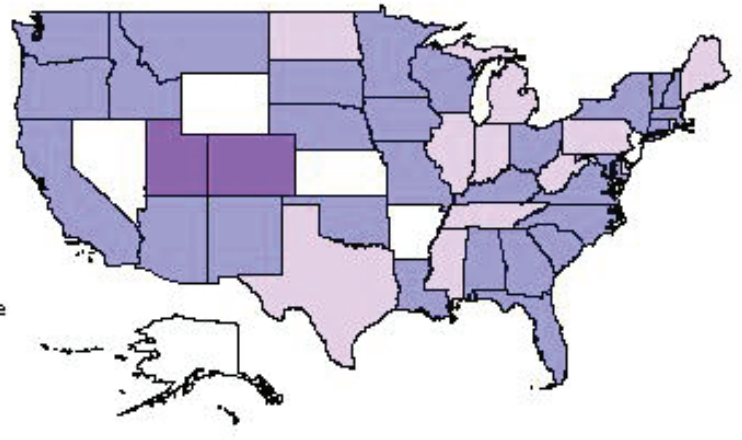

Female, corrected, 1990

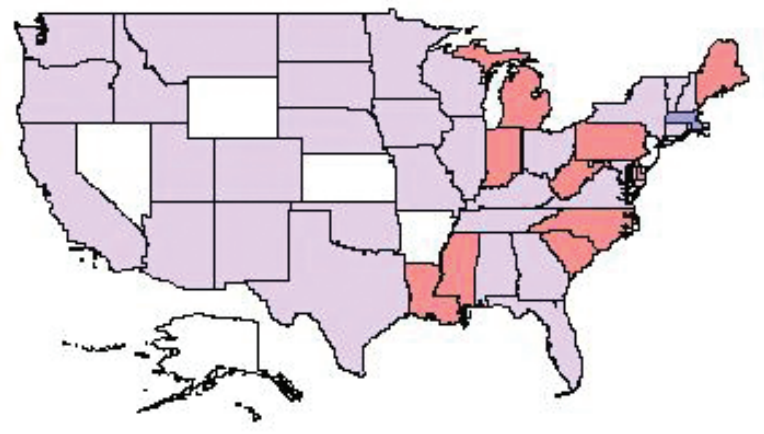

Male, corrected, 2000

(b)

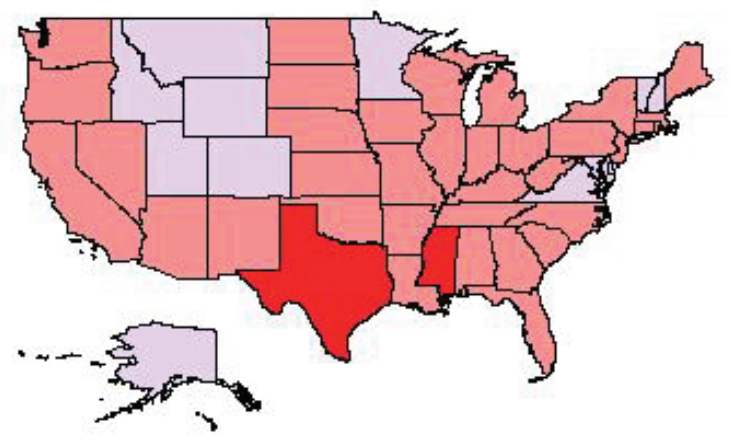

Female, corrected, 2000

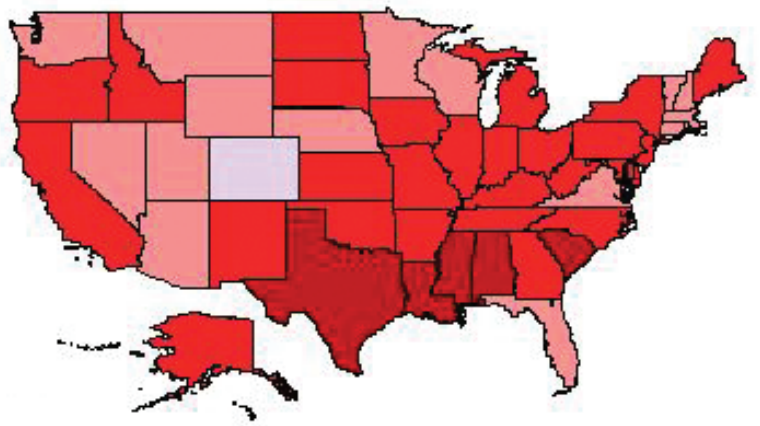




\section{DISCUSSION}

\section{Principal findings}

Our results provide the first estimates of the levels and trends in state-level obesity in the USA, corrected for bias in self-reported height and weight. Using national population-level comparisons of self-reported and measured weight and height in the USA, we found that, compared to the 'gold standard' of measured health examination survey, on average, women underreported their weight, but men did not. Young and middle-aged ( $<65$ years) men overreported their height more than women of the same age. In the older age groups, over-reporting of height was similar in men and women. A population-level bias in self-reported weight was greater in telephone interviews than in inperson interviews. Except in older people, height was overreported with a greater bias in telephone interviews than in-person interviews with a follow-up examination. In 2000, using the corrected weight and height, Mississippi (31\%) and Texas (30\%) had the highest prevalence of obesity for men and Texas (37\%), Louisiana (37\%), Mississippi (37\%), District of Columbia (37\%), Alabama (37\%), and South Carolina (36\%) for women.

\section{Comparison with other obesity surveillance studies}

Previous reports on bias in self-reported weight and height $^{9-14}$ had all been based on individual-level data. As a result, these works could not examine two factors important for population-level monitoring: first, individuallevel misreporting caused by absence of measurement subsequent/previous to the interview and by the mode of self-report, and second, differential participation based on the survey mode. Previous reports on state-level obesity levels and trends in the USA were based on the BRFSS, 7,8 which uses telephone surveys, and hence significantly underestimates true obesity as seen in Figure 5.

\section{Strengths and weaknesses of the study}

Our results are subject to uncertainty because there may be systemic variation in misreporting across states and social groups, or over time, for example because of differences and changes in social values related to weight and height. If such a variation exists, it would create heterogeneity in the relationship used for correction (Figure 3), not detectable in our data. Repeated cross-sections in 1988-1994 and 1999-2002 did not indicate a systemic change in this relationship during the analysis period. The evidence on the role of race and education as determinants of bias was also not conclusive (data not shown), and smaller than the effects of age and sex.

Self-reported data on weight and height are the only feasible option for large population surveys that are both nationally and sub-nationally representative and conducted on a regular basis (e.g. annual) in most nations (a small number of industrialized countries like the UK and Japan conduct annual measurements, but most are not subnationally representative). The choice for health researchers and practitioners is therefore between using self-reported weight and height, which are known to be subject to large bias, or relying on a correction algorithm like the one presented in this work that reduces bias, albeit with some uncertainty.

\section{Conclusions and future research}

The ideal correction to self-reported height and weight data would be from a study in which subjects initially report their height and weight in telephone interviews with the expectation that they would not be measured later; but they are, in fact, subsequently measured (e.g. by asking to attend a medical examination at the end of the telephone interview). In such a study, the results (see Figure 3) could be further divided by age or other socio-demographic factors. This would allow researchers to examine the interactions of such factors and actual height/weight as determinants of bias, which was not possible in our analysis. Such a study, to the best of our knowledge, does not currently exist but would be an ideal addition to the BRFSS. Even if such a validation study were implemented, the problem of selection would persist - both in the initial survey and in the validation phase-because some people who misreported their height and weight in the telephone survey would not agree to subsequent measurement. In the absence of such an ideal validation study, the method used in our analysis - correcting self-reported data using height and weight from telephone surveys on those from health examinations surveys - is the best available option for unbiased estimates of the levels and trends in state-level obesity in the USA.

Acknowledgments ME and CJLM designed the study and the figures. HM, ME, SS, and SVH conducted the analysis. ME wrote the manuscript with input from other authors. ME will act as the guarantor for the work.

This research was supported by a cooperative agreement from the Centers for Disease Control and Prevention (CDC) through the Association of Schools of Public Health (ASPH) Grant No. U36/CCU300430-23, and by the National Institute on Aging (Grant No. PO1-AG17625). The contents of this article are solely the responsibility of the authors and do not necessarily represent the official views of CDC or ASPH.

Competing interests None. 


\section{REFERENCES}

1 Ezzati M, Lopez AD, Rodgers A, Vander Hoorn S, Murray CJL. Comparative Risk Assessment Collaborative Group. Selected major risk factors and global and regional burden of disease. Lancet 2002;360:1347-60

2 Pelletier DL, Rahn M. Trends in body mass index in developing countries. Food Nutrition Bull 1998;19:223-39

3 James WTP, Leach R, Mhurchu CN, et al. Overweight and obesity (high body mass index). In: Ezzati M, Lopez AD, Rodgers A, Murray CJL, eds. Comparative Quantification of Health Risks: Global and Regional Burden of Disease Attributable to Selected Major Risk Factors. Geneva: World Health Organization, 2004:497-596

4 Mokdad A, Marks J, Stroup D, Gerberding J. Actual causes of death in the United States, 2000. JAMA 2004;291:1238-45

5 Flegal KM, Graubard BI, Williamson DF, Gail MH. Excess deaths associated with underweight, overweight, and obesity. JAMA 2005;293:1861-7

6 Flegal KM, Carroll MD, Ogden CL, Johnson CL. Prevalence and trends in obesity among US adults, 1999-2000. JAMA 2002; 288:1723-7

7 Mokdad AH, Serdula MK, Dietz WH, Bowman BA, Marks JS, Koplan JP. The spread of the obesity epidemic in the United States, 19911998. JAMA 1999;282:1519-22

8 Mokdad A, Bowman B, Ford E, Vinicor F, Marks J, Koplan J. The continuing epidemics of obesity and diabetes in the United States. JAMA 2001;286:1195-200

9 Engstrom JL, Paterson SA, Doherty A, Trabulsi M, Speer KL. Accuracy of self-reported height and weight in women: an integrative review of the literature. J Midwifery Women's Health 2003;48:338-45
10 Villanueva EV. The validity of self-reported weight in US adults: a population based cross-sectional study. BMC Public Health 2001;1:11

11 Black DR, Taylor AM, Coster DC. Accuracy of self-reported body weight: Stepped Approach Model component assessment. Health Educ Res 1998;13:301-7

12 Jeffery RW. Bias in reported body weight as a function of education, occupation, health and weight concern. Addict Behav 1996;21:217-22

13 Schmidt MI, Duncan BB, Tavares M, Polanczyk CA, Pellanda L, Zimmer PM. Validity of self-reported weight - a study of urban Brazilian adults. Rev Saude Publica 1993;27:271-6

14 Zhang J, Feldblum PJ, Fortney JA. The validity of self-reported height and weight in perimenopausal women. Am J Public Health 1993; 83:1052-3

15 National Center for Health Statistics. Plan and operation of the Third National Health and Nutrition Examination Survey, 1988-94. Vital Health Stat 1994;32:1-407

16 Kuczmarski MF, Kuczmarski RJ, Najjar M. Effects of age on validity of self-reported height, weight, and body mass index: findings from the Third National Health and Nutrition Examination Survey, 1988-1994. J Am Diet Assoc 2001;101:28-34; quiz 35-6

17 Korn EL, Graubard BI. Analysis of Health Surveys. New York: John Wiley, 1999

18 Objectives and design of the Behavioral Risk Factor Surveillance System. Proceedings of The Section On Survey Methods, American Statistical Association National Meeting, 1998, Dallas, Texas. Alexandria: ASA, 1998

19 Remington PL, Smith MY, Williamson DF, Anda RF, Gentry EM, Hogelin CG. Design, characteristics, and usefulness of state-based behavioral risk factor surveillance:1981-1987. Public Health Rep 1988; 103:366-75 